\title{
Continuity of care is in the eye of the beholder
}

\section{Michael Wright}

CONTINUITY OF CARE is increasingly in the news. A recent systematic review found strong evidence that continuity of care, defined as repeated contact between an individual patient and a doctor, was associated with decreased patient mortality. The review included 22 studies from nine countries in three continents. Of these studies, 18 reported that increased continuity of care from one doctor was associated with significant reductions in mortality. ${ }^{1}$

Providing continuity of care has long been one of The Royal Australian College of General Practitioners' standards and is considered a core component of highperforming healthcare systems. But are we clear in what we mean by continuity of care?

In 1980, Barbara Starfield spoke of the 'continuous confusion' that existed in attempts to understand and measure continuity of care. ${ }^{2}$ Since then, numerous authors have sought to define continuity of care. The most widely used typology - developed by Canadian authors in an article titled 'Defusing the confusion' proposed continuity of care within three main dimensions: ${ }^{3}$

- the traditional notion of continuity of care considered within the terms of the doctor-patient relationship (relational continuity of care)

- the availability of appropriate information to allow safe, coherent care (informational continuity)

- consistency of care delivery (management continuity).

Australia has an ageing population that is increasingly living with chronic diseases, and a general practice workforce whose members are increasingly working in larger practices. These changes may be reducing the ability of patients to obtain ongoing care with one provider. As our practices become larger and the complexity of the care we provide grows, it is timely to reflect on how patients experience (or fail to experience) continuity of care. Australians can attend multiple general practitioners (GPs) and multiple practices for their care, whereas some other countries encourage continuity of care through practice enrolment, patient lists or practice registration. ${ }^{4}$

This issue of Australian Journal of General Practice (AJGP) provides an opportunity to review the breadth of continuity of care research in Australia, clarify what we mean by continuity of care and see where continuity of care fits in modern general practice. Most commonly, continuity of care refers to the proportion of care with one doctor, as defined in the recently published systematic review by Pereira Gray et al; ${ }^{1}$ however, a small body of research is evaluating continuity of care at a practice level (site continuity of care), 5,6 which may be relevant to Australian general practice.

The challenges of measuring and understanding continuity of care at a practice level are discussed by Jackson and Ball $^{7}$ and highlight the need for clarity in our research when describing continuity of care. The article by Hambleton outlines the potential benefits of improved electronic communication on information flows to patients, between GPs and through the broader health system. ${ }^{8}$ The rollout of My Health Record is likely to improve informational continuity between different parts of the health system; however, the effects on relational continuity are less clear.

Other articles discuss the role of continuity of care in improving medication management, ${ }^{9}$ and reflect on how we might achieve the benefits of a strong doctor-patient relationship while retaining patient choice and rapid access to general practice care when needed within our current system. ${ }^{10}$

So have we cleared the confusion? Perhaps not, but hopefully the articles in this issue of AJGP provide insights into some of the challenges Australian general practice faces and how improving continuity of care (in all its dimensions) may provide some solutions.

\section{Author}

Michael Wright MBBS, MSc, FRACGP,

$\mathrm{PhD}$ candidate, Centre for Health Economics Research and Evaluation, University of Technology Sydney, NSW

\section{References}

1. Pereira Gray DJ, Sidaway-Lee K, White E, Thorne A, Evans PH. Continuity of care with doctors - A matter of life and death? A systematic review of continuity of care and mortality. BMJ Open 2018;8(6): e021161. doi: 10.1136/bmjopen2017-021161.

2. Starfield B. Continuous confusion? Am J Public Health 1980;70(2):117-19.

3. Reid RJ, Haggerty J, McKendry R. Defusing the confusion: Concepts and measures of continuity of healthcare. Ottawa: Canadian Health Services Research Foundation, 2002.

4. Kalucy $L$, Katterl R, Jackson-Bowers $E$, Hordacre A-L. Models of patient enrolment. PHCRIS policy issue review. Adelaide: Primary Health Care Research Information Service, 2009.

5. Roos LL, Roos NP, Gilbert P, Nicol JP. Continuity of care: Does it contribute to quality of care? Med Care 1980;18(2):174-84.

6. Atlas SJ, Grant RW, Ferris TG, Chang Y, Barry MJ. Patient-physician connectedness and quality of primary care. Ann Intern Med 2009;150(5):325-35.

7. Jackson C, Ball L. Continuity of care: Vital, but how do we measure and promote it? Aust J Gen Pract 2018;47(10):662-64.

8. Hambleton S. A glimpse of 21 st century care. Aust J Gen Pract 2018;47(10):670-73.

9. Stasinopoulos J, Bell JS, Manski-Nankervis J, Hogan M, Jenkin P, Sluggett J. Medication management of type 2 diabetes in residential aged care. Aust J Gen Pract 2018;47(10):675-81.

10. Wright M, Mainous AG. Can continuity of care in primary care be sustained in the modern health system? Aust J Gen Pract 2018;47(10):667-69. 